\title{
La anticoagulación oral es superior al clopidogrel más aspirina en pacientes con fibrilación auricular
}

ACTIVE Writing Group. Lancet. 2006;367(9526):1903-12.

\section{Objetivo}

Evaluar si el clopidogrel más aspirina no era inferior a la anticoagulación oral para la prevención de eventos vasculares en pacientes con fibrilación auricular (FA).

\section{Diseño}

Ensayo clínico controlado aleatorizado.

\section{Lugar}

Estudio multicéntrico internacional.

\section{Pacientes}

Se incluyeron 6706 pacientes con FA y ${ }^{3} 1$ factor de riesgo de accidente cerebrovascular (ACV): ${ }^{3} 75$ años; hipertensión arterial; ACV previo, accidente isquémico transitorio (AIT) o embolismo sistémico (excluyendo ACV); fracción de eyección ventricular izquierda $<45 \%$; arteriopatía periférica; 55-74 años con diabetes o enfermedad coronaria. Se excluyeron pacientes con contraindicación a las drogas evaluadas, válvula protésica; úlcera péptica dentro de los 6 meses previos; historia de hemorragia cerebral; trombocitopenia o estenosis mitral. Se aleatorizaron a recibir anticoagulación oral (RIN de 2-3; $n=3371$ ) o clopidogrel (75 mg/día) + aspirina (75$100 \mathrm{mg} / \mathrm{día} ; \mathrm{n}=3335$ ). La FA era permanente en la mayoría ( $>2$ años en el 59\%); ACV/AIT en $15 \%$ y $28 \%$ tenían enfermedad coronaria.

\section{Medición de resultados principales}

El resultado primario fue ACV, embolismo sistémico (excluyendo ACV), infarto de miocardio (IM) o muerte vascular. Un comité ciego también evaluó las hemorragias. El análisis fue por intención de tratar.

\section{Resultados principales}

El estudio se detuvo tempranamente debido a la clara evidencia de superioridad de la anticoagulación oral (Tabla 1) con un NND* anual de 52 (IC 95\% 17-45). La anticoagulación previno más todo tipo de ACV, en especial los menos serios. Los pacientes anticoagulados estuvieron $21 \%$ del tiempo con un $\mathrm{RIN}<2$.

Los pacientes asignados a anticoagulación, ya anticoagulados antes de ingresar al estudio, comparados al grupo clopidogrel-aspirina tuvieron, además de mejor control y menos abandonos, una tendencia hacia mayor reducción en los eventos vasculares (RR $1.50,95 \% \mathrm{Cl} 1.19-1.89$ ) y un riesgo significativamente más bajos de sangrado mayor (1.30; 0.94-1.79) que los pacientes no anticoagulados a la entrada al estudio $(1.27,0.85-1.89$ y $0.59,0.32-1.08$, respectivamente).

Tabla 1. Resultados primarios y secundarios

\begin{tabular}{|c|c|c|c|c|c|c|}
\hline \multirow[b]{2}{*}{ Resultados } & \multicolumn{2}{|c|}{$\begin{array}{c}\text { Clopidogrel + } \\
\text { aspirina }\end{array}$} & \multicolumn{2}{|c|}{$\begin{array}{l}\text { Anticoagulación } \\
\text { oral }\end{array}$} & \multicolumn{2}{|c|}{$\begin{array}{l}\text { Clopidogrel+aspirina vs } \\
\text { Anticoagulación oral }\end{array}$} \\
\hline & $n$ & Biesgo anual & $n$ & Riesgo anual & RR (1C95\%) & $p$ \\
\hline Resultado primario & 234 & $5,60 \%$ & 165 & $3,93 \%$ & $1,44(1,18-1,76)$ & 0,0003 \\
\hline Embolismo (no ACV) & 18 & $0,43 \%$ & 4 & $0,10 \%$ & $4,66(1,58-13,8)$ & 0,005 \\
\hline Infarto de miocardio & 36 & $0,86 \%$ & 23 & $0,55 \%$ & $1,58(0,94-2,67)$ & 0,09 \\
\hline ACV & 100 & $2,39 \%$ & 59 & $1,40 \%$ & $1,72(1,24-2,37)$ & 0,001 \\
\hline Mortalidad total & 159 & $3,80 \%$ & 158 & $3,76 \%$ & $1,01(0,81-1,26)$ & 0,91 \\
\hline Muerte vascular & 120 & $2,87 \%$ & 106 & $2,52 \%$ & $1,14(0,88-1,48)$ & 0,34 \\
\hline Hemorragia mayor (sev. o fatal) & 101 & $2,42 \%$ & 93 & $2,21 \%$ & $1,10(0,83-1,45)$ & 0,53 \\
\hline Hemorragia menor & 568 & $13,58 \%$ & 481 & $11,45 \%$ & $1,23(1,09-1,39)$ & 0,0009 \\
\hline $\begin{array}{l}\text { Resultado primario o } \\
\text { hemorragia mayor }\end{array}$ & 316 & $7,56 \%$ & 229 & $5,45 \%$ & $1,41 \quad(1,19-1,67)$ & $<0,0001$ \\
\hline $\begin{array}{l}\text { Resultado primario, } \\
\text { hemorragia mayor o muerte }\end{array}$ & 348 & $8,32 \%$ & 271 & $6,45 \%$ & $1,31(1,12-1,54)$ & 0,0008 \\
\hline
\end{tabular}

\section{Conclusiones}

La anticoagulación oral es superior al clopidogrel más aspirina para la prevención de eventos vasculares en los pacientes con FA con alto riesgo de $\mathrm{ACV}$, sobre todo en aquéllos que ya tomaban anticoagulantes.

Fuente de financiamiento: Sanofi-Aventis and Bristol-Myers Squibb.

\section{Comentario}

La anticoagulación oral reduce $2 / 3$ el riesgo de ACV comparado con placebo ${ }^{1}$ y $45 \%$ vs. aspirina ${ }^{2}$.

Sin embargo es necesario el control a largo plazo ya que aumenta un $70 \%$ el riesgo de sangrados mayores comparado con aspirina ${ }^{2}$ y muchos pacientes no logran una anticoagulación óptima. El presente estudio que evaluaba la combinación de clopidogrel y aspirina debió detenerse tempranamente debido a la clara superioridad de la anticoagulación oral.

Esto se debe a la prevención de eventos embólicos, en especial ACV, por la formación de trombos cardíacos para los cuales la anticoagulación es particularmente efectiva. En contra de lo esperado en otros estudios ${ }^{3}$, la combinación de clopidogrel y aspirina no fue más eficaz previniendo IM que la anticoagulación, también efectiva en pacientes con cardiopatía isquémica ${ }^{4}$. La falta de superioridad de la anticoagulación sobre la mortalidad total podría explicarse por su relativa incapacidad para prevenir los eventos más severos. Las hemorragias menores fueron menos frecuentes con anticoagulación pero las hemorragias fatales ligeramente más comunes. Las tasas de eventos fueron menores que en los estudios de los '901 debido al menor riesgo de los pacientes y al mejor tratamien-

Agustín Ciapponi [ Servicio de Medicina Familiar y Comunitaria. Hospital Italiano de Buenos Aires. ] to, incluyendo un control más estrecho del RIN. Aún así la diferen-

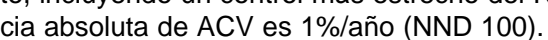

Debido a una mayor exposición previa a la anticoagulación que a clopidogrel-aspirina podría existir un sesgo al seleccionar más pacientes con buena respuesta y mejor adherencia a la primera intervención. Esto se vio claramente en los sangrados mayores que con el antecedente de anticoagulación previa, los sangrados eran menos frecuentes en el grupo anticoagulación y lo inverso sin dicho antecedente. La extrapolación de los resultados del estudio a pacientes sin tratamiento anticoagulante previo es menos clara.

\section{Conclusión del comentador}

En pacientes previamente tratados con anticoagulantes, la anticoagulación es claramente superior a la combinación clopidogrel-aspirina aun considerando las hemorragias y las muertes además de los eventos vasculares. No hay razón para cambiar el tratamiento antitrombótico estándar de la FA fundamentalmente basado en la anticoagulación.

Ver glosario*

Recibido el 25/9/06 y aceptado el 29/10/06

Ciapponi A. La anticoagulación oral es superior al clopidogrel más aspirina en pacientes con fibrilación auricular. Evid. Actual. Práct. Ambul. 9(6); 166 NovDic. 2006. ACTIVE Writing Group on behalf of the ACTIVE Investigators; Connolly S, Pogue J, Hart R, Pfeffer M, Hohnloser S, Chrolavicius S, Pfeffer M, Hohnloser S, Yusuf S. Clopidogrel plus aspirin versus oral anticoagulation for atrial fibrillation in the Atrial fibrillation Clopidogrel Trial with Hohnloser S, Yusuf S. Clopidogrel plus aspirin versus oral anticoagulation for atrial fibrillation in the Atrial fibrillation
Irbesartan for prevention of Vascular Events (ACTIVE W): a randomised controlled trial. Lancet. 2006;367(9526):1903-12.

Referencia

1. Atrial Fibrillation I. Risk factors for stroke and efficacy of antithrombotic therapy in atrial fibrillation: analysis of pooled data from five randomized controlled trials. Arch Intern Med 1994:1449-57.

2. C van Walraven, RG Hart and DE Singer et al., Oral anticoagulants vs aspirin in nonvalvular atrial fibrillation: an individual patient meta-analysis, JAMA 288 (2002), pp. 2441-8. 3. The Clopidogrel in Unstable angina to prevent Recurrent Events (CURE) trial investigators, Effects of clopidogrel in addition to aspirin in patients with acute coronary syndromes without ST-segment elevation, N Engl J Med 2001;345:494-502.

4. M Hurlen, M Abdelnoor, P Smith, J Erikssen and H Arnesen, Warfarin, aspirin, or both after myocardial infarction, N Engl J Med 347 (2002), pp. 969-974. 